\title{
Mode locking of semiconductor laser with curved waveguide and passive mode expander
}

Cite as: Appl. Phys. Lett. 82, 322 (2003); https://doi.org/10.1063/1.1539277

Submitted: 09 September 2002 . Accepted: 26 November 2002 . Published Online: 15 January 2003

C. A. Williamson, M. J. Adams, A. D. Ellis, and A. Borghesani

Applied Physics Reviews

Now accepting original research 


\title{
Mode locking of semiconductor laser with curved waveguide and passive mode expander
}

\author{
C. A. Williamson a) and M. J. Adams \\ Department of Electronic Systems Engineering, University of Essex, Wivenhoe Park, Colchester, \\ CO4 3SQ United Kingdom
}

\author{
A. D. Ellis and A. Borghesani \\ Corning Research Centre, Adastral Park, Martlesham Heath, Ipswich, Suffolk, IP5 3RE United Kingdom
}

(Received 9 September 2002; accepted 26 November 2002)

\begin{abstract}
Active mode locking is reported for a $1.55 \mu \mathrm{m}$ semiconductor laser with a curved waveguide and passive mode expander, placed in a wavelength tunable external cavity. One facet with a very low reflectivity of $8 \times 10^{-6}$ is achieved through a curved active region that tapers into an underlying passive waveguide, thus expanding the mode to give reduced divergence. $10 \mathrm{GHz}$ pulses of $3.1 \mathrm{ps}$ duration have been generated, with a linewidth of $0.81 \mathrm{~nm}$. (C) 2003 American Institute of Physics. [DOI: 10.1063/1.1539277]
\end{abstract}

A variety of applications ranging from optical communications to radar systems require picosecond optical pulses at high repetition rates. Active mode locking is one technique used to generate such pulses from semiconductor lasers by applying a rf drive current at a frequency matching the roundtrip of the laser cavity. The cavity lengths of conventional semiconductor lasers are typically a few hundreds of microns, leading to roundtrip frequencies of the order of 100 GHz. Placing a device in an external cavity system is one option to reduce these roundtrip frequencies to the order of gigahertz for applications in present-day optical communications systems. Mode locking of external cavity lasers is a well-established technique, with picosecond and subpicosecond pulses being achieved., ${ }^{1,2}$

When using an external cavity configuration it is desirable to have high reflectivity at the output facet of the semiconductor laser while maintaining very low reflectivity at its inner facet to couple to the external cavity. With imperfect antireflection coatings at this facet, multiple pulses are generated at the semiconductor laser roundtrip frequency. ${ }^{1}$ These additional pulses are undesirable for most applications, and also deplete the power of the primary pulse. An inner facet reflectivity of $10^{-4}$ is often quoted ${ }^{3}$ as a minimum value for satisfactory mode locking operation, but facet reflectivities of the order of $10^{-6}$ are needed to more completely suppress the formation of subpulses. ${ }^{4}$

Semiconductor lasers with angled facets have a reduced reflectivity due to the reduction in overlap between the reflected light and the guided mode, and have been mode locked in the past. ${ }^{5}$ So too have devices with a curved or "bent" waveguide to realize both high and low reflectivity facets on the same device. ${ }^{6}$ There has also been mode locking of a curved waveguide laser with a tapered active region; ${ }^{7}$ in this case the active region increased in width to give higher powers due to multimode operation.

This letter presents the active mode locking of a 1.55 $\mu \mathrm{m}$ semiconductor laser with a curved waveguide to reduce the reflectivity at only one facet, and an active region that

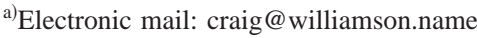

tapers down towards this facet to allow the guided mode to expand into an underlying passive waveguide. ${ }^{8}$ This further reduces the reflectivity due to the smaller divergence of the expanded mode, while also enhancing the coupling efficiency and relaxing alignment tolerances out into the external cavity. ${ }^{9}$ The resulting inner facet reflectivity has been reported $^{8}$ to be $8 \times 10^{-6}$, while the outer facet maintains a conventional reflectivity of approximately 0.3 . The features of very low reflectivity at only one facet and an expanded mode waveguide make the device ideal for use in an external cavity setup.

The semiconductor structure is approximately $1 \mathrm{~mm}$ in length and has a buried heterostructure design. Figure 1(a) shows its active layer, with eight quantum wells of $\mathrm{In}_{0.84} \mathrm{Ga}_{0.16} \mathrm{As}_{0.68} \mathrm{P}_{0.32}$ at $70 \AA$ thickness, strained $+1 \%$ with respect to the InP substrate. The barriers are $140-\AA$-thick 1.3 $\mu \mathrm{m}$ InGaAsP with a strain of $-0.5 \%$, and at the top and bottom of the active region is a $100-\AA$-thick separate confinement heterostructure $(\mathrm{SCH})$ of the same composition and strain as the barriers.

In Fig. 1(b) a schematic top-view diagram of the chip is shown, displaying the curved and tapered active layer. The chip begins with a $300 \mu \mathrm{m}$ untapered active section of 1.2 $\mu \mathrm{m}$ width that curves to a final angle of $10^{\circ}$. Next a $450 \mu \mathrm{m}$ active section tapers down to a width of $0.15 \mu \mathrm{m}$, and the guided mode expands into the underlying passive waveguide of $7 \mu \mathrm{m}$ width. The dimensions of the passive guide are designed to increase the laser spot size to match that of an optical fiber, ${ }^{10}$ while the active taper length is optimized for minimal loss between the two sections. ${ }^{11}$ The final section of $250 \mu \mathrm{m}$ is purely passive and allows the expanded mode to stabilize.

The device is situated in an external cavity formed between the conventional facet of the semiconductor laser and a moveable grating. The grating selects a small group of modes for amplification through the semiconductor and subsequent mode locking, and may be tuned through a wavelength range of approximately $30 \mathrm{~nm}$. An antireflectioncoated ball lens is used to couple the light from the low reflectivity facet out into the external cavity, and a tapered fiber is brought up to the outer semiconductor facet to moni- 
(a)

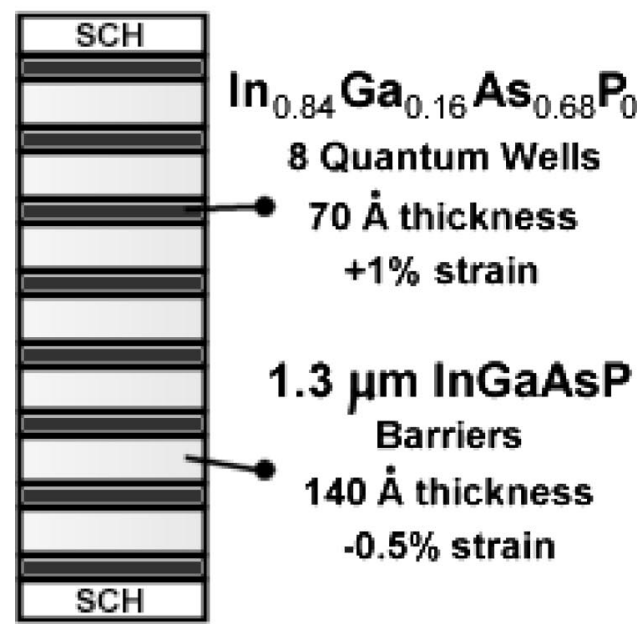

(b)

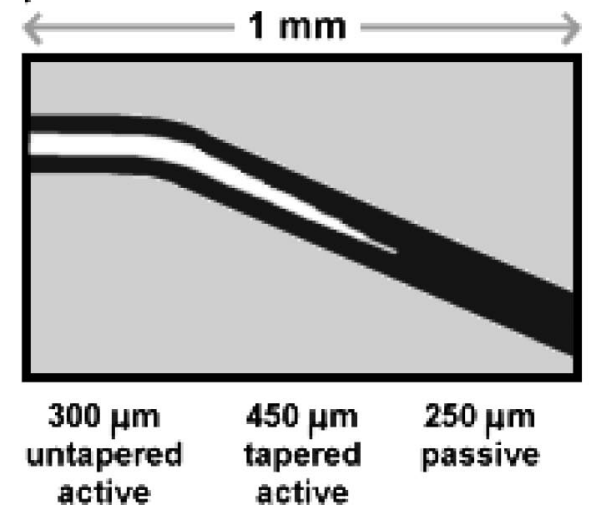

FIG. 1. Structure of the semiconductor laser used in the active mode locking experiments. (a) Composition of the active region. (b) Top-view schematic showing the curved and tapered active region.

tor the light output from the external cavity system. ${ }^{12}$

A batch of five identical devices has been mode locked using a cavity frequency of $2.5 \mathrm{GHz}$, corresponding to an external cavity length of approximately $5.6 \mathrm{~cm}$. The setup was mode locked in fundamental operation at $2.5 \mathrm{GHz}$, and also in harmonic operation at $10 \mathrm{GHz}$. The results obtained with each of the devices were comparable within the experimental uncertainties, and the results of a typical device will now be discussed. rf powers were the maximum available from the equipment, with $35 \mathrm{dBm}$ at $2.5 \mathrm{GHz}$ (estimated to be around $170 \mathrm{~mA}$ ), and $30 \mathrm{dBm}$ at $10 \mathrm{GHz}$ (approximately $56 \mathrm{~mA})$. A higher dc bias $(50 \mathrm{~mA})$ was required for satisfactory mode locking performance at $10 \mathrm{GHz}$ to compensate for the reduced rf power, as compared with $30 \mathrm{~mA}$ for $2.5 \mathrm{GHz}$.

Due to the linear red chirp created in the active mode locking process by the variation in refractive index with carrier density, ${ }^{13}$ it is possible to compress pulses by the introduction of a length of optical fiber with negative dispersion. The compression of pulses has not been optimized, but results with a $100 \mathrm{~m}$ length of fiber with a dispersion of -90.6 $\mathrm{ps} / \mathrm{nm} / \mathrm{km}$ at $1550 \mathrm{~nm}$ are given to demonstrate that the pulses can indeed be compressed.

Figure 2(a) shows autocorrelation functions of the mode locked pulses obtained at $10 \mathrm{GHz}$. Without compression,
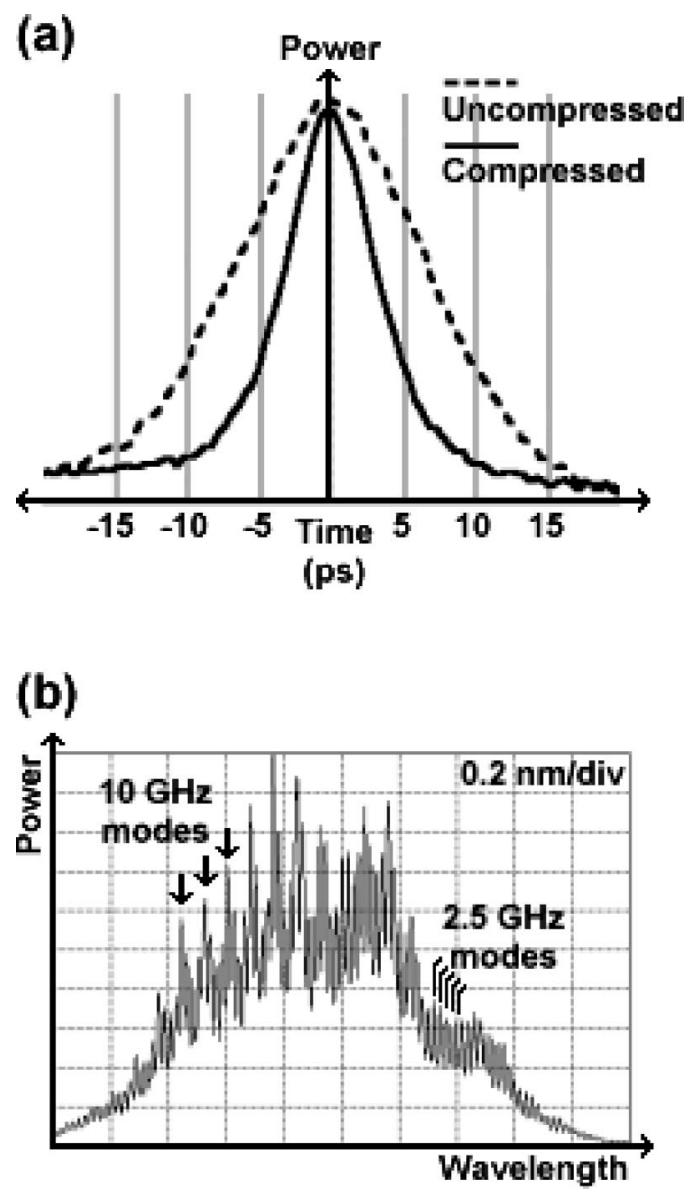

FIG. 2. Results of active mode locking at $10 \mathrm{GHz}$. (a) Autocorrelation functions for uncompressed and compressed pulses, relating to symmetric two-sided exponential pulses of pulse widths 6.1 and 3.1 ps, respectively. (b) Spectrum of the mode locked pulses showing mode spacing due to the 10 $\mathrm{GHz}$ driving frequency and the $2.5 \mathrm{GHz}$ cavity length.

single pulses with an autocorrelation function full width at half maximum (FWHM) of 14.8 ps were obtained, without any visible subpulses. Through the nonoptimized compression, this was reduced to 7.5 ps. The autocorrelation functions most closely match those given by symmetric twosided exponential actual pulse shapes. Under this assumption, the actual pulse widths were 6.1 and $3.1 \mathrm{ps}$ for the uncompressed and compressed pulses, respectively.

Figure 2(b) shows the spectrum obtained from these pulses. The smaller mode spacing corresponds to $2.5 \mathrm{GHz}$ as determined by the cavity length, while the spacing of the sharper mode spikes relates to the $10 \mathrm{GHz}$ driving rf frequency. The FWHM linewidth was measured to be $0.81 \mathrm{~nm}$, giving time-bandwidth products that were 4.4 and 2.2 times the transform limit for the uncompressed and compressed pulses, respectively. Coupled average powers were limited to the microwatt range due to the manual fiber alignment at the output facet. The picosecond pulsewidths, subnanometer linewidth, and wavelength tuning capability makes this setup a candidate source for a multiwavelength optical time division multiplexed (OTDM) communications system.

When mode locking at $2.5 \mathrm{GHz}$, there were no subpulses visible once again. An uncompressed autocorrelation width of $12.5 \mathrm{ps}$ was obtained, which was compressed to $6.9 \mathrm{ps}$ with use of the dispersion compensating fiber. Again matching to symmetric two-sided exponential pulse shapes, this 
gives actual pulse widths of 5.2 and 2.9 ps for the uncompressed and compressed pulses, respectively. With a linewidth of $1.09 \mathrm{~nm}$, the time-bandwidth products of these pulses were 5.0 and 2.8 times the transform limit, and average output powers were once more in the microwatt range.

In summary, a $1.55 \mu \mathrm{m}$ semiconductor laser with a curved waveguide and passive mode expander has been actively mode locked at 2.5 and $10 \mathrm{GHz}$ frequencies. Through its conventional reflectivity outer facet, very low reflectivity inner facet, and expanded mode output with low divergence, this device is well suited to such an external cavity setup. Nonoptimally compressed pulses of 2.9 ps at $2.5 \mathrm{GHz}$ and $3.1 \mathrm{ps}$ at $10 \mathrm{GHz}$ were obtained, with linewidths of 1.09 and $0.81 \mathrm{~nm}$, respectively. Together with its wavelength tuning capability, these features make the setup a candidate source for a multiwavelength OTDM communications system.

The authors are grateful to the device fabrication group at Corning Research Centre for supplying devices. One of the authors (C.W.) is indebted to EPSRC and Corning Research Centre for financial support.
${ }^{1}$ J. E. Bowers, P. A. Morton, A. Mar, and S. W. Corzine, IEEE J. Quantum Electron. 25, 1426 (1989).

${ }^{2}$ J. Yu, M. Schell, M. Schulze, and D. Bimberg, Appl. Phys. Lett. 65, 2395 (1994).

${ }^{3}$ P. Vasil'ev, Ultrafast Laser Diodes (Artech House, Boston, 1995), p. 111. ${ }^{4}$ M. Schell, A. G. Weber, E. Schöll, and D. Bimberg, IEEE J. Quantum Electron. 27, 1661 (1991)

${ }^{5}$ J. Chen, W. Sibbett, and J. I. Vukusic, Electron. Lett. 18, 426 (1982).

${ }^{6}$ T. Yilmaz, C. M. DePriest, and P. J. Delfyett, Jr., Electron. Lett. 37, 1338 (2001).

${ }^{7}$ R. Helkey, W. X. Zou, A. Mar, D. B. Young, and J. Browers, IEEE Trans. Electron Devices 40, 2107 (1993).

${ }^{8}$ I. F. Lealman, A. E. Kelly, L. J. Rivers, S. D. Perrin, and R. Moore, Electron. Lett. 34, 2247 (1998).

${ }^{9}$ P. Doussiere, P. Garabedian, C. Graver, E. Derouin, E. Gaumont-Goarin, G. Michaud, and R. Meilleur, Appl. Phys. Lett. 64, 539 (1994).

${ }^{10}$ I. F. Lealman, L. J. Rivers, M. J. Harlow, and S. D. Perrin, Electron. Lett. 30, 1685 (1994).

${ }^{11}$ I. F. Lealman, L. J. Rivers, M. J. Harlow, S. D. Perrin, and M. J. Robertson, Electron. Lett. 30, 857 (1994).

${ }^{12}$ A. J. Lowery and I. W. Marshall, Electron. Lett. 26, 104 (1990).

${ }^{13}$ M. Schell, J. Yu, M. Tsuchiya, and T. Kamiya, Appl. Phys. Lett. 67, 1797 (1995). 